\title{
TEMA CENTRAL
}
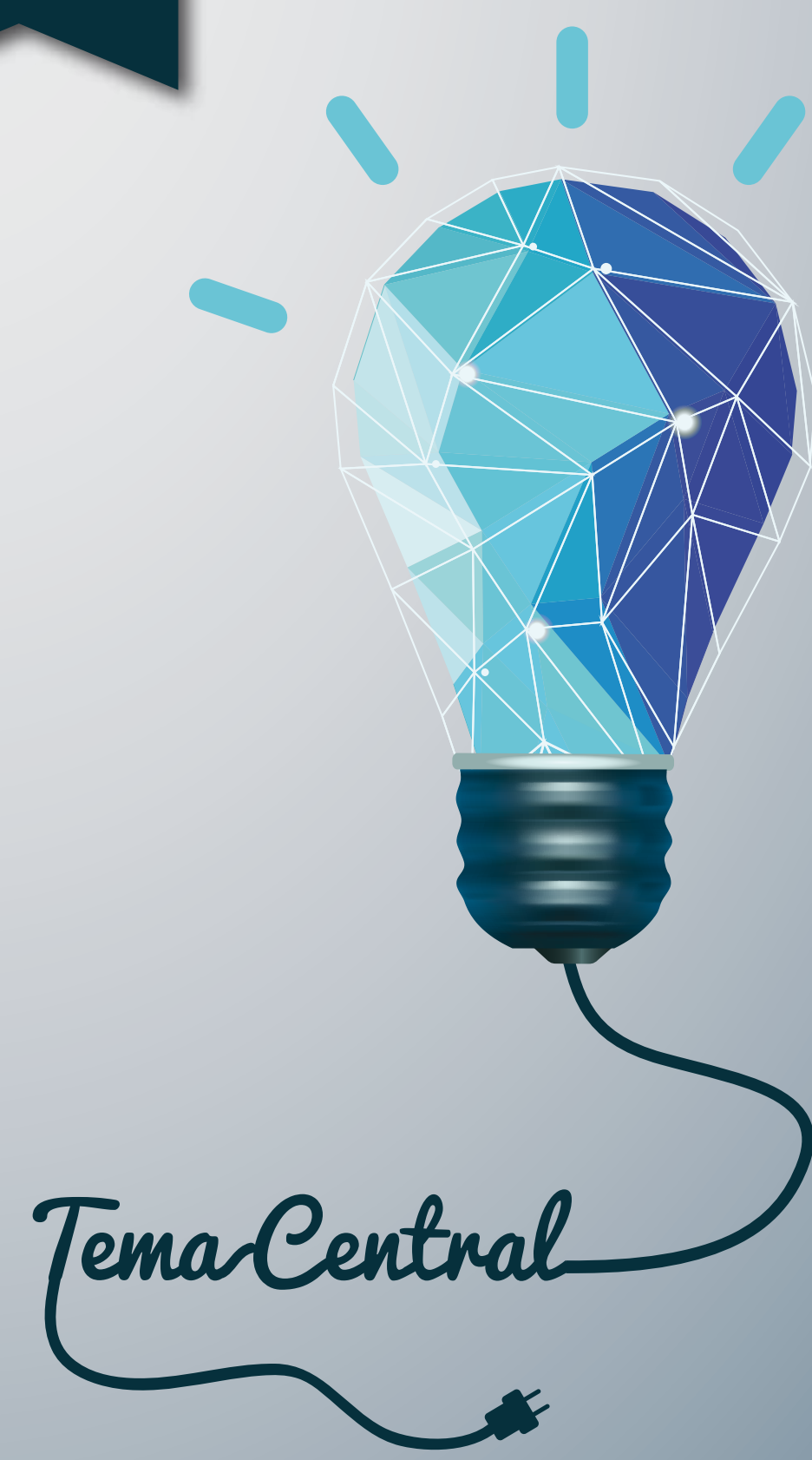



\section{Retos en la escritura de artículos científicos de estudiantes de posgrado de la UNAH}

Nitzia Janine Vásquez ${ }^{1}$

\section{INTRODUCCIÓN}

El presente artículo refleja la experiencia que desde la DICYP hemos capitalizado producto de la relación directa con la edición de las revistas "Ciencia y Tecnología" y "Portal de la Ciencia", medios a través de los cuales los profesores-investigadores y los estudiantes de la UNAH, publican sus artículos que, en general se proponen dar a conocer los resultados de sus investigaciones.

El abordaje del presente artículo está centrado en las habilidades y destrezas de los estudiantes de posgrado en la redacción de artículos científicos para ser publicados en revistas indexadas, especialmente en las revistas a cargo de la DICYP.

Es de hacer notar que las instituciones de educación superior a nivel internacional demanda investigadores, no solamente con una sólida formación académica, sino que además cuenten con un respaldo en investigación, usualmente validado a través de publicaciones en revistas indexadas. Publicar artículos científicos se ha convertido en una necesidad, especialmente para los estudiantes de posgrado.

Las universidades están dando mucha atención a procesos de potenciación de competencias cognitivas, procedimentales y de precisión en el uso del lenguaje para escribir artículos con claridad, precisión y brevedad.

Con frecuencia al redactar artículos científicos se cometen errores, que generalmente son producto de la falta de formación en la redacción de este tipo particular de texto, así como problemas en la adecuación, o su exposición del contenido bajo los parámetros demandados por las revistas.

1 Jefa del Departamento de Documentación e Información de la DYCYP, UNAH: investigacionunah.informa.nv@gmail.com 


\section{Comenzando a escribir}

El artículo científico es un texto escrito, generalmente publicado en medios especializados, con el propósito de comunicar a la comunidad científica los resultados de un trabajo de investigación, realizado mediante la aplicación del método científico, según las características desarrolladas en cada disciplina. (Venegas ,2005)

¿Y qué es lo que necesita un estudiante investigador para poder publicar? ¿Se requiere de destrezas o habilidades especiales? ¿Por qué resulta tan complejo? Para el estudiante de posgrado, quien ha desarrollado progresivamente su tesis, la redacción de un artículo representa un momento crucial pues, es una prueba fuerte de capacidades cognitivas, habilidades y destrezas que deben manifestarse en un artículo que deberá pasar por un surtidor de expertos relativos al tema en criterios sólidos sobre jerarquización de información, valoración de contenidos, incongruencias estilísticas, debilidades gramaticales, de puntuación, acentuación y vocabulario. No es suficiente desarrollar las investigaciones científicas, también es importante escribirlas y publicarlas.

\section{Los problemas típicos de escritura}

Según Jerz (2000), existen varios tipos de problemas típicos que enfrentan los estudiantes universitarios en la escritura técnica: la falta de revisión, el mal manejo del espacio en blanco, inconsistencia en el uso de siglas y abreviaciones y la escasa jerarquización de la información. A su vez, Feldman, Anderson y Mangurian (2001) revisan una serie de cuatro problemas a los que se enfrentan los estudiantes que ingresan a la universidad, con relación con la escritura: las debilidades en la lógica, incongruencias estilísticas relativas a la disciplina o a la audiencia, el plagio y una serie de debilidades mecánicas de gramática y vocabulario, son los problemas para los cuales los autores van sugiriendo soluciones específicas. Los errores más comunes de los artículos que se presentan a la DICYP corresponden a debilidades mecánicas de gramática, como por ejemplo, errores en ortografía, en puntuación, en conjugación de verbos (género y número en los nombres), uso inadecuado del léxico e incluso la falta de coherencia en la estructura de los párrafos.

Es aconsejable, que los estudiantes al escribir un artículo científico tomen en cuenta tres aspectos generales para una escritura apropiada. 
1. Conocer los aspectos atinentes a la construcción de la estructura general de un artículo científico, tratando de explicar la información de manera clara, breve, neutral y adecuadamente referenciada para facilitar la lectura del artículo.

2. Tener dominio disciplinar (tanto teórico como metodológico) junto con el meta conocimiento disciplinar compuesto por los aspectos culturales señalados por Becher (2001). El meta conocimiento se refiere a la mejora de las habilidades de aprendizaje en la formación estudiantil, por ejemplo el desarrollo de habilidades para comprensión de textos. Se asume que todo estudiante de posgrado debe poseer una serie de conocimientos especializados en un área de investigación específica para poder escribir un artículo con contenidos originales y novedosos para su publicación.

3. Manejar con propiedad las características relativas del género, al proceso de su producción y consumo (envío, edición, publicación).

La Dirección de Investigación Científica consciente del grado de complejidad que enfrentan los estudiantes de posgrado para escribir con propiedad artículos científicos, que luego deben ser publicadas en revistas científicas indexadas, está llevando a cabo desde hace algunos años jornadas de capacitación a fin de potenciar las capacidades de los estudiantes en referencia, y que puedan con solvencia profesional redactar sus artículos con marca de calidad, en estos procesos de habilitación para redactar de acuerdo a normas estandarizadas se incluyen temas relacionados a la estructura de un artículo científico, las normas para citar y referenciar y demás elementos clave de la escritura de artículos que se presentan a la DICYP. En materia de plagio se imparte un curso denominado Trabajos académicos, referencias bibliográficas y plagio para que los investigadores conozcan la importancia de evitar el plagio y el software disponible en la UNAH para detectar el mismo.

Todos los elementos en mención representan un desafío para los estudiantes de posgrado. En muchos casos, el desconocimiento o la falta de reforzamiento conceptual en estas dimensiones, así como la no identificación de las normas específicas de publicación, conllevan errores en las estructuras generales del artículo científico. En consecuencia, es pertinente comenzar a elaborar un plan general de redacción que intente responder una serie de preguntas como: ¿Cuál es el propósito de la investigación? ¿Cuál es su relevancia en el campo de conocimiento? ¿Qué se hizo metodológicamente? ¿Cómo lo hizo? ¿Qué resultados se encontraron? ¿Cuál es el significado del experimento o proyecto? Cuando se revise, se pueden colocar preguntas y respuestas necesarias para mejorar el primer ejercicio. Este proceso es sumamente 
útil para poder elegir lo que se debe decir y omitir lo que no se debe decir.

Resulta práctico cuando ya se tiene una estructura del artículo, porque también se puede ir destinando un número de páginas aproximado. Por ejemplo, la introducción puede ser de unos cuantos párrafos que no sobrepasen la longitud de una hoja. De esta manera, se empleará menos tiempo escribiendo, ya que la estructura está definida y se pueden dejar los detalles para después. La escritura debe ser clara y concisa, procurando expresar las ideas con simplicidad. Se puede auxiliar preguntándose lo siguiente: ¿El artículo está en coherencia con el mensaje que se quiere transmitir? ¿El mensaje que se transmite concuerda con la finalidad de la revista en donde se pretende publicar?

A continuación se procede con la revisión del manuscrito procurando tener en cuenta lo siguiente: ¿Cumple con las reglas básicas de ortografía y gramática? ¿Los datos de los cuadros y gráficos están verificados? ¿Cuál es el estilo permitido para el uso de cuadros y gráficos? ¿Se están respetando los criterios de publicación establecidos por la revista indexada? ¿Respecto al título, se ha revisado la puntuación, uso de verbos, uso de números, de acuerdo a lo aceptado en la revista? ¿Las referencias bibliográficas coinciden con las citas incluidas en el texto y viceversa? ¿Acaso el resumen condensa de manera apropiada el mensaje a trasmitir? Deberá leerse el articulo muchas veces para poder identificar lo que hace falta. Se recomienda solicitar ayuda a un colega para que también lo lea y aporte detalles que el autor quizás haya olvidado mencionar.

Con el objetivo de que un grupo de estudiantes aprenda a escribir un artículo científico se pueden intentar varias prácticas:

1. Los estudiantes preparan un documento a partir de la rescritura de un artículo ya publicado. De esta manera, el artículo les sirve como modelo para poder redactar objetivos, formular adecuadamente las preguntas y poder comprender los aspectos metodológicos (Lubomir, 2007).

2. Los estudiantes universitarios de carreras científicas pueden redactar un trabajo de investigación basándose en la integración de diversas estrategias de lectura y escritura. La estrategia de análisis, cuando se refiere a la literatura científica, significa entender como otros contribuyeron a contestar la hipótesis, y validar la evidencia presentada de acuerdo a la metodología de investigación utilizada. La estrategia de síntesis, significa crear una nueva idea al combinar la información presentada por otras fuentes, e incluso reorganizar su propia percepción del 
tema para poder contestar la hipótesis. Estas estrategias les permiten fortalecer las destrezas en redacción científica. (Kolikant, Gatchell, Hirsch y Linsenmeier ,2006).

3. El sistema informático Labwrite es ideal para la escritura de informes de laboratorio. Consiste en una plantilla de uso electrónico que les permite a los estudiantes iniciar su proyecto contestando las siguientes preguntas: ¿Cuál es la relevancia científica de este proyecto? ¿Cuáles son los objetivos? ¿Cuál es el propósito del proyecto? ¿Cuál es la hipótesis? La plantilla les guiará paso a paso para ir describiendo la metodología, los resultados, la introducción, la discusión, las conclusiones, el resumen, el título y las referencias bibliográficas. Este sistema ayuda a los estudiantes a construir su propio informe de laboratorio al momento de contestar preguntas clave y les permite tener una mejor concepción de la estructura de un informe científico (Ferzli, Carter y Wiebe, 2005).

\section{Desafíos para los estudiantes de posgrado}

A continuación se presentan una serie de desafíos sobre los cuales es importante trabajar para corregir algunos problemas que se manifiestan en la escritura de artículos científicos. En primer lugar, resulta necesario reforzar algunas habilidades en los elementos ortográficos básicos y puntuales sobre los que usualmente suelen detectarse algunas deficiencias, como por ejemplo el uso inadecuado de mayúsculas y errores de acentuación de palabras. En efecto, los problemas asociados a mala puntuación, errores ortográficos o problemas en la estructuración de oraciones representan un nivel básico en el cual se puede intervenir.

En segundo lugar, un desafío más apremiante radica en los problemas que podrían ubicarse en la macrorredacción, es decir, en la estructura misma de los textos producidos para espacios académicos especializados. La formación en grado es esencial para comenzar a resolver este desafío, ya que es en este etapa en donde los estudiantes tienen que identificar las características de su disciplina para tener una noción del lenguaje a utilizar en la redacción de textos académicos, especialmente conocer la forma de expresión y las reglas con las cuales los pares se comunican.

Un tercer desafío, también articulado con los dos precedentes, es la formación investigativa y su forma de plasmarla. Como se sabe, el método de investigación no significa lo mismo que el método de exposición de los resultados de investigación. En 
muchos casos, se presentan inadecuaciones que remiten a la estructura misma del trabajo de investigación, en donde no existe una coherencia entre el planteamiento, objetivos y resultados de investigación, por tanto, más que un problema de redacción es un problema de fondo de la investigación en general. En muchos casos, se reporta prácticamente al final de la investigación que algunos propósitos del estudio no fueron plenamente cubiertos, por lo cual el artículo puede mostrar algunas incongruencias. Este problema remite especialmente a la formación investigativa de los alumnos de posgrado, por tanto, una tentativa de solución es comenzar intervenciones orientadas a que desde la escritura de la propuesta de redacción los estudiantes comiencen a tener una formación en redacción científica.

La Dirección de Investigación Científica y Posgrados reconoce las debilidades y fortalezas que actualmente presentan los artículos científicos de estudiantes de posgrado. Para fomentar la escritura de artículos se han incluido una cláusula especial en cada contrato de beca de investigación en la cual se solicita la publicación de un artículo por cada beca otorgada. En el caso de becas otorgadas a estudiantes de posgrado se ha destinado una revista, Portal de la Ciencia, para poder brindarles este espacio de publicación a los investigadores.

\section{Respecto a las Normas Académicas de la UNAH}

La lectura y escritura científica es una parte indispensable de cada investigador. La literatura científica es el canal de comunicación más importante ya que les permite a los investigadores el poder transmitir la sabiduría de la comunidad científica. Uno de los objetivos de educación superior es introducir a los estudiantes en el proceso de investigación científica y en la lectura y escritura de artículos para enriquecer su vida profesional. Es por esta razón que la Universidad Autónoma de Honduras, a partir del año 2015 ha agregado un requisito adicional para los estudiantes que egresan de programas de maestría y doctorados. Las Normas Académicas de la UNAH, en su Capítulo IV, sobre las funciones fundamentales de la UNAH, en su sección B sobre la investigación científica, en su Artículo 25 señala que:

La investigación en las maestrías académicas y en los doctorados tiene como propósito principal el mostrar la madurez intelectual y el dominio del método científico que ha logrado el estudiante de posgrado. El proceso y los resultados de la investigación de estos posgrados se organizan en tesis doctorales y tesis de maestría, generando durante su proceso de producción por lo menos tres (3) artículos publicables, que serán 
valorados considerando los niveles de complejidad y profundidad de la maestría y del doctorado.

Por años, la tesis ha sido el único requisito de graduación, ya que es un documento fundamental en donde se plasma la investigación de los estudiantes de maestría y doctorado. La normativa actual representa un verdadero reto para los programas de maestría que no están fomentando la publicación de artículos o los que no están impulsando la investigación. Por lo tanto, los programas de maestría deberán preguntarse lo siguiente: ¿Será suficiente la cantidad de asesores en cada maestría para poder revisar estos artículos? ¿Cuáles son las deficiencias de los estudiantes en materia de escritura científica? ¿Cómo se puede fortalecer la investigación para cumplir con la normativa actual?

La Dirección de Investigación Científica y Posgrados apoya la normativa vigente al fomentar la creación de nuevas revistas por facultad. Cada año se hace una revisión de las revistas científicas para evaluar la calidad y proponer estrategias para mejorarlas. Actualmente, la UNAH cuenta con las siguientes revistas científicas:

1. Ciencia y Tecnología (Dirección de Investigación Científica y Posgrado)

2. Portal de la Ciencia (Dirección de Investigación Científica y Posgrado)

3. Población y Desarrollo (Maestría en Demografía)

4. Revista de Ciencias Sociales (Facultad de Ciencias Sociales)

5. Ciencias Espaciales (Facultad de Ciencias Espaciales)

6. Acta Pediátrica Hondureña (Escuela Universitaria de las Ciencias de la Salud)

7. Revista Científica de la Escuela Universitaria de las Ciencias de la Salud

8. Revista de la Facultad de Ciencias Médicas

9. Economía y Administración (Instituto de Investigaciones Económicas y Sociales)

10. Revista de la Escuela de Física

11. Revista Arte y Cultura (Centro de Arte y Cultura)

12. Innova (Dirección de Innovación Educativa)

13. Revista de Derecho (Instituto de Investigaciones Jurídicas)

14. Perspectivas del Desarrollo (Doctorado en Ciencias Sociales)

Estas revistas han sido creadas para la publicación de artículos científicos que cumplen con estándares internacionales de calidad. Los estudiantes de posgrado podrán publicar en estas revistas siempre y cuando cumplan con las instrucciones a los autores, que aparecen en cada revista. Sin embargo, en algunas facultades se necesitará de espacios adicionales para poder publicar investigaciones y para ello se 
deberán crear comités editoriales compuestos por profesores de la misma facultad e investigadores externos.

Sin duda alguna, es un desafío para los estudiantes que puedan construir los artículos con la calidad pertinente requerida para su publicación en una revista científica de renombre. Muchas veces se conoce el procedimiento de cómo organizar el artículo, pero se desconoce la forma en cómo cuidar la calidad requerida. Es por ello que se deben evaluar las revistas nacionales conforme a parámetros internacionales de revisión y aceptación de manuscritos. De esta manera, se mejora la calidad de la revista y se permite el acceso de la misma a una mayor cantidad de portales de revistas indexadas en línea. Esto permite un mayor índice de citación, ya que un artículo escrito por un alumno de un posgrado de medicina en la UNAH puede ser leído y citado por un estudiante de un posgrado similar en otra universidad de América Latina, o sea, a mayor visibilidad, mayor índice de citación.

La Dirección de Investigación Científica continuará impulsando la investigación científica en todos los niveles de formación de estudiantes. A través de los cursos de Redacción científica y Trabajos académicos, referencias bibliográficas y plagio continuará orientando a los investigadores en el las destrezas requeridas para la publicación de artículos de calidad. En conjunto con INASP, la Biblioteca de la UNAH y la UNAH se continuará elevando el nivel de las revistas indexadas en portales internacionales, a través de talleres diseñados para los miembros de comités editoriales de revistas. En la medida de que se mejoren las revistas en forma y contenido, existirá la posibilidad de pertenecer a mayor índices de revistas indexadas internacionales. Ha llegado el momento de elevar los estándares, fijar nuevas metas y apoyar la investigación científica desde el alma máter. 


\section{BIBLIOGRAFÍA}

Becher, T. (2001). Tribus y territorios académicos: La indagación intelectual y las culturas de las disciplinas. Barcelona: Gedisa.

Feldman, Susannah; Anderson, V. \& Mangurian, Luz. (2001). Teaching effective scientific writing. Journal of College Science Teaching, 30(7), 446-450.

Ferzli, Miriam; Carter, Michael \& Wiebe, Eric. (2005). LabWrite. Journal of College Science Teaching, 35(3), 31-33.

Jerz, Dennis. (2000). Top five problems for writing academic essays. Recuperado de: http://jerz.setonhill.edu/writing/technical/resume/tips.htm

Kolikant, Yifat; Gatchell, David; Hirsch, Penny \& Linsenmeier, Robert. (2006). A cognitive-apprenticeship-inspired instructional approach for teaching scientific writing and reading. Journal of College Science Teaching, 36(3), 20-25.

Lubomir, Tomaska. (2007). Teaching how to prepare a manuscript by means of rewriting published scientific papers. Genetics. Doi: 10.1534/genetics.106.066217

Universidad Nacional Autónoma de Honduras. (2015). Normas Académicas de la Universidad Nacional Autónoma de Honduras. Tegucigalpa: Editorial Universitaria.

Venegas, Rene. (2005). Las relaciones léxico-semánticas en artículos de investigación científica: Una aproximación desde el análisis semántico latente. Revista Signos. Doi:10.4067/S0718-09342006000100004 
